with bilateral talipes, or any child with the spastic form of cerebral palsy, should be examined particularly carefully for subluxation of the hip. Again, it should be a routine to check the hearing of any child who is late in speaking, or who speaks indistinctly for his age, or whose parents express doubts whether he can hear properly. To this extent it is an advantage to have a risk register which draws the attention of doctors to the need to examine and follow certain children with particular care. It might be more useful, however, to add to the child-welfare clinic's chart a small section drawing attention to the special ways in which a particular child is at risk. For instance, if it were recorded that the father had congenital deafness, anyone seeing the chart would know he should take special notice of the child's hearing. Part of the routine examination of any infant in a welfare clinic, general practice, or hospital, must include a brief developmental assessment, measurement of the maximum head circumference and its relation to the weight of the baby, inspection of the back for a congenital dermal sinus needing treatment to prevent meningitis, examination of the hips for subluxation, of the urine for phenylpyruvic acid, and after three or four months a rapid test of the baby's hearingall procedures which can be done in a busy clinic in a very few minutes. But that is a different matter from suggesting that local authorities should spend time and money in keeping a special register.

A risk register has some possible disadvantages apart from the time and expense of maintaining it. Mothers may worry when told that their children are on the register. The proper examination of other children not on the register may be neglected, though experience has shown they may also have a fairly high incidence of abnormalities. And the risk might be exaggerated when assessing an individual child. This applies particularly to a child who is being assessed for adoption. Tragedies can occur if children are said to be unsuitable for adoption because of "risk factors" in their past. The physician has to assess children on their merits; even if a mother is a certified mental defective her baby may be entirely normal and eminently suitable for adoption. Though doctors should know the "risk factors," it is wise not to overemphasize their importance.

Whether a risk register is used or not, there is a danger of forgetting that a child with one handicap is at risk of having another. When paying particular attention to the handicap already found, such as congenital heart disease, it is easy to overlook another, such as congenital dislocation of the hip or deafness.

\section{Human Vibriosis}

Vibrio fetus is an important cause of infectious abortion in ungulates, a group which includes cattle and sheep. The organism has a specific predilection for the pregnant uterus, where it causes suppuration and necrosis of the membranes, with foetal death. Protracted carrier states often follow infection, when the organism may be remarkably localized to the cervical canal or circumscribed areas of the uterus. Antibody may be present in the cervical mucus but there is commonly no systemic response. ${ }^{1}$ Closely related organisms also occur in cattle as commensals (Vibrio bubulus) and as the cause of one variety of infectious diarrhoea (Vibrio jejuni). In cattle the disease appears to be transmitted venereally. The infected bull harbours the organism indefinitely in the testis, and it has frequently been recovered from the semen of apparently healthy animals.

The similar vibrio in sheep appears to be culturally and antigenically distinct from the bovine type, and to differ in its mode of transmission. It appears not to be spread venereally but by ingestion and has for this reason been called Vibrio fetus var. intestinalis. Other animal species are susceptible, and it has been suggested that birds may play a part in transporting the disease from one herd or flock to another. ${ }^{2}$

In contrast with the long. latent infection of animals, with abortion as its only dramatic symptom, is an uncommon severe infection in man. It has been increasingly recognized over the last few years. This week in the B.M.F. at page 283 Dr. W. D. White and at page 287 Dr. J. H. Darrell, Mr. B. C. Farrell, and Dr. Rosemary A. Mulligan now add the first cases recorded in Great Britain. In man the disease is not particularly an infection of the gravid uterus, as it is in animals. Among the 34 cases collected by M. D. Willis and W. J. Austin ${ }^{3}$ eight involved pregnant women or newborn babies, but the remaining 26 were in men. In the newborn baby the central nervous system is commonly attacked, and the organism may be recovered from the cerebrospinal fluid. A. N. Eden ${ }^{4}$ has drawn attention to the similarity between many of the features of this infection and listeriosis and has suggested that it may be a commoner cause of perinatal mortality than has so far been recognized.

The severity of the human disease is illustrated by C. Kilo and colleagues ${ }^{3}$; of 30 patients 5 died. As in the British patients now described, there has been nothing in the previous cases to suggest the diagnosis clinically, though in retrospect there are seen to be some features in common. The diagnosis has, for practical purposes, invariably been made by blood culture, and the hazards in the path of the bacteriologist unwittingly called on to recognize this particular pathogen are well described in the present accounts. Primary isolation requires relatively prolonged microaerophilic incubation, and growth on subculture even in optimum conditions may be so slight as to be overlooked. Once isolated, there is always the danger that so bizarre an organism will be discarded as a contaminant. It is possible that the bacteriological diagnosis may in due course be facilitated by fluorescent-antibody methods. ${ }^{6}$

But it is not only the diagnosis which is difficult; the epidemiology of the disease is obscure and its treatment unsettled. Infection with an animal pathogen naturally invites the speculation that those concerned with animal husbandry are likely to be most at risk, but this does not appear to be so. In several cases the onset of the disease has been related to tooth extraction, and this has focused attention on the vibrios which normally live in the mouth. They, and the vibrios of the normal vagina, appear to belong to a single species, Vibrio sputorum. It is distinct from the pathogenic Vibrio fetus but is antigenically related to it and

\footnotetext{
1 Manclark, C. R., and Pickett, M. J., F. Path. Bact., 1965, 90, 627.

2 Frank, F. W., Waldhalm, D. C., Meinershagen, W. A., and Scrivner L. H., F. Amer. vet. med. Ass., 1965, 147, 1313 .

"Willis, M. D., and Austin, W. J., Amer. f.' Dis. Child., 1966, 112, 459.

- Eden, A. N., F. Pediat., 1966, 68, 297.

Silo, C., Hagemann, P. O., and Marzi, J., Amer. F. Med., 1965, 38, 962.

- Belden, E. L., and Robertstad, G. W., Amer. F. vet. Res., 1965, 26,

' Loesche, W. J., Gibbons, R. J., and Socransky, S. S., f. Bact., 1965,

89, 1109. vet. Res., 1964, 25, 1295.
} 
may be responsible for part of the confusion over the presence of vibrio antibodies in human serum. ${ }^{7}$

Analogy with cattle suggests that the human disease might be venereally transmitted, but such attempts as have been made to recover the organism from human semen have failed. $^{3}$ Biochemically, the vibrios infecting man appear to resemble those of sheep more than those of cattle, ${ }^{7}$ and it is possible that this similarity extends to their being transmitted by mouth rather than by sexual intercourse. It is strikingand this is again illustrated by the British cases-how infrequently an immediate source of infection has been identified and how commonly the patients have already been suffering from some other condition such as heart disease, cirrhosis, leukaemia, or chronic anaemia. ${ }^{5}$ This certainly favours the idea that the latent disease, as in cattle, may be more common than is suspected and become activated, perhaps years after the original infection, by some other disease. ${ }^{4}$

Authors have generally been divided on whether streptomycin or tetracycline is the treatment of choice, and perhaps at the moment the suggestion of Dr. Darrell and his colleagues is the right one-that a combination of the two should be given. On the other hand, those physicians blessed with a laboratory well enough equipped to make the diagnosis for them might do better to rely on it to sort out the optimum treatment by in-vitro methods.

\section{Injuries from Vomiting}

In 1724 Boerhaave described spontaneous rupture of the oesophagus associated with a violent episode of vomiting. ${ }^{1}$ This occurred in a Baron Wassenaar, Grand Admiral of the Dutch Fleet, who was a notorious glutton and who practised postprandial autoemesis. By 1946 N. R. Barrett ${ }^{2}$ was able to review some fifty cases of spontaneous oesophageal rupture which ended in death. He suggested that prompt diagnosis and early intervention might alter this gloomy outlook, and fulfilled his forecast two years later by reporting the first successful repair. ${ }^{3}$

Clinically the diagnosis is suggested by violent epigastric, substernal, or back pain which immediately follows an episode of vomiting. The patient may even complain of a tearing sensation in the chest. There may be accompanying shock, cyanosis, and dyspnoea. Often upper abdominal guarding and tenderness are present. Surgical emphysema is a common finding, and there is often clinical or radiological evidence of a hydropneumothorax. Differential diagnosis includes a perforated duodenal ulcer (the commonest error), coronary thrombosis, pancreatitis, and dissecting abdominal aneurysm. A chest $x$-ray which shows mediastinal emphysema and air or fluid in the pleural cavity is the best guide to this comparatively unusual diagnosis. The tear is usually situated on the left posterior aspect of the oesophagus immediately above the diaphragm and is longitudinal. Though spontaneous recovery is known, surgical repair as soon as possible after diagnosis offers the best chance of survival.

Some two hundred years after Boerhaave's monograph G. K. Mallory and S. Weiss ${ }^{4}$ reported on 15 patients who developed massive haematemesis after prolonged alcoholic indulgence. Each had had preceding bloodless vomiting. Four of these patients came to necropsy and each showed multiple mucosal fissures at the cardia, their long axis being in line with the oesophagus. Microscopical examination showed that these lacerations extended into the muscularis. An experiment on a cadaver showed that gastric distension with the oesophagus occluded at the level of the cardia produced similar changes.

It has now been recognized ${ }^{5}$ that this syndrome of mucosal tearing at the cardia is by no means invariably related to alcoholism and indeed may not necessarily be preceded by vomiting. It may be associated with a hiatus hernia and is particularly prone to occur in elderly patients. The typical tears have been observed on many occasions at gastroscopy, allowing accurate diagnosis to be made preoperatively. Continued bleeding may demand laparotomy, and it is important for the surgeon to be aware of this lesion, for it can be seen at operation only by direct observation through an adequate gastrotomy. Bleeding is controlled by suturing the tears. ${ }^{6}$ ?

In recent years oesophageal rupture and mucosal tears at the cardia have been related to each other by their essentially similar mechanism of production-a sudden rise in intragastric or intraoesophageal pressure, usually produced by violent vomiting or retching, but on occasions due to abdominal trauma by a blunt instrument, defaecation, childbirth, weight-lifting, compressed air, and so on. J. Lion-Cachet ${ }^{8}$ distended fresh cadaver stomachs while observing the cardiac region by means of a cystoscope introduced through the oesophagus. The muscle wall of the stomach was more distensible than its mucosa, which gave way with an audible "phut," resulting in the type of tear described by Mallory and Weiss." This also occurred when the lower extremity of the oesophagus was distended. The rest of the oesophagus behaved in a different manner. The outer muscle coat reached its limit first and then the mucosa ruptured through it, producing a tear of the Boerhaave type. ${ }^{1}$

All grades of injury from mucosal laceration to complete rupture of the oesophagus and the stomach have been described. Recently the spectrum has been completed by N.W. Thompson and his colleagues, who, in reviewing nine patients with postemetic injuries, include two patients with intramural "dissections" of the stomach and oesophagus, which have not previously been reported. It seems reasonable, then, that different grades of postemetic injury occur. There may be a superficial mucosal tear of either the oesophagus or stomach which results in little or no haemorrhage. If this is more extensive, massive arterial bleeding results. A greater force may result in rupture of the wall or, as Thompson and colleagues report, an intramural injury. There have been reports of patients in whom haemorrhage first occurred and was followed hours or days later by rupture of the oesophagus. At operation or necropsy combinations of both mucosal tearing and full-thickness rupture have been seen. Pain associated with haematemesis should alert the clinician to the possibility of potential or actual rupture.

\footnotetext{
1 Aird, I., Companion in Surgical Studies, 1957, 2nd ed. London.

2 Barrett, N. R., Thorax, 1946, 1, 48.

- Brit. F. Surg., 1948, 35, 216.

- Mallory, G. K., and Weiss, S., Amer. F. med. Sci., 1929, 178, 506.

s Atkinson, M., Bottrill, M. B., Edwards, A. T., Mitchell, W. M., Peet, B. G., and Williams, R. E., Gut, 1961, 2, 1.

- Scott, N. M., and Newton, D. E., Amer. 9. dig. Dis., 1958, 3, 464.

' Small, A. B., and Ellis, P. R., New Engl. F. Med., 1958, 258, 285.

- Lion-Cachet, J., Brit. f. Surg., 1963, 50, 985. - Thompson, N. W., Ernst, C. B., and Fry, W. J., Amer. F. Surg., 1967,
113, 13.
} 